\title{
Intra-Vitreous Injections of Bevacizumab in Choroidal Neovascularization Complicating Best Vitelliform Macular Dystrophy: Case Report of a Child
}

\author{
Elkhoyaali A*, J eddou I, Zerrouk R, Khanaouchi \\ N, Mouzari Y, Reda K and Oubaaz A \\ Ophthalmology Department, Mohamed V University, \\ Military Training Hospital Mohamed V, Rabat, Morocco \\ *Corresponding author: Adil Elkhoyaali, Department \\ of Ophtalmology, Mohamed V University, Military \\ Hospital Mohamed V, Rabat, Morroco
}

Received: May 25, 2021; Accepted: J une 16, 2021; Published: J une 23, 2021

\begin{abstract}
A 15-year-old child followed for Best vitelliform macular dystrophy presented to the clinic with an abrupt visual impairment of his left eye. Fundus examination showed bilateral vitelliform lesions, with serous retinal detachment and adjacent retinal hemorrhage in the left eye. Fluorescein angiography and optical coherence tomography confirmed the diagnosis of type-2 choroidal neovascularization complicating the Best disease. The juxtafoveal location of the choroidal neovascularization prompted us to realize monthly intra-vitreous injections of bevacizumab. After the fourth injection, we observed visual and anatomical improvement that remained stable after a 12-month follow-up. It's important to look for complications in front of a sudden decrease in visual acuity in Best disease.
\end{abstract}

Keywords: Best disease; Macular dystrophy; Choroidal neovascularization; Intra-vitreous injections; Bevacizumab

\section{Introduction}

Best Vitelliform Macular Dystrophy (BVMD) is among the most common genetic macular degeneration typically occurring bilaterally with juvenile-onset [1]. The development of choroidal neovascularization $(\mathrm{CNV})$ is an uncommon complication with no proven effective treatment modality at this time.

\section{Case Presentation}

We report the case of a child followed for BVMD who presented to the ophthalmology clinic for a sudden visual impairment that happened to be caused by choroidal neovascularization.

\section{Results}

We report the case of a 15-year-old male diagnosed with BVMD seven years earlier based on electrophysiological examination (perturbed electro-oculography and subnormal Arden ratio). Visual acuity remained stable at $20 / 32$ in both eyes during five years, then the patient was lost to follow-up for 30 months, before attending the ophthalmology clinic for a bilateral rapidly progressive decrease of vision and metamorphopsia, mostly in the left eye.

Best-Corrected Visual Acuity (BCVA) was 20/40 OD and 20/160 OS. Intraocular pressure was normal. Fundus examination revealed bilateral foveal fibrotic vitelliform lesion, surrounded by a serous retinal detachment (SRD) and an inferior macular microhemorrhage in the left eye (Figure 1).

Left eye fluorescein angiogram showed a lesion that hyperfluoresces in the early stages due to a pigment epithelial window defect, maintains well-demarcated borders, and leaks late suggestive of an active subfoveolar choroidal neovascularization. Inferior to this lesion, we found a localized hypofluorescence area caused by the masking effect of subretinal haemorrhage (Figure 2).

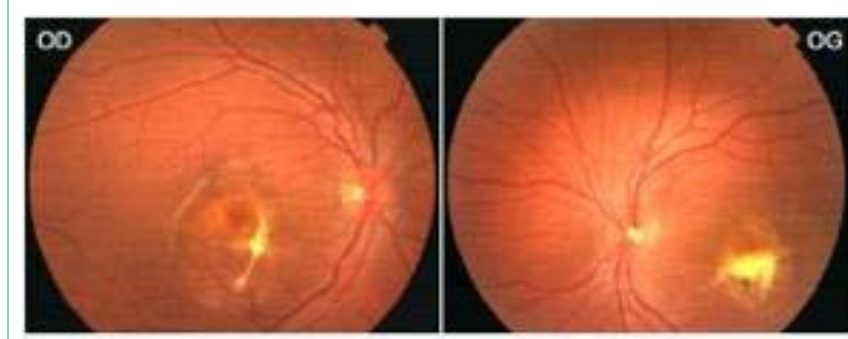

Figure 1: Fundus photography of both eyes: oval macular dystrophy with an irregular greyish appearance in the left eye associated with retinal serous detachment and sub-foveal intraretinal haemorrhage.

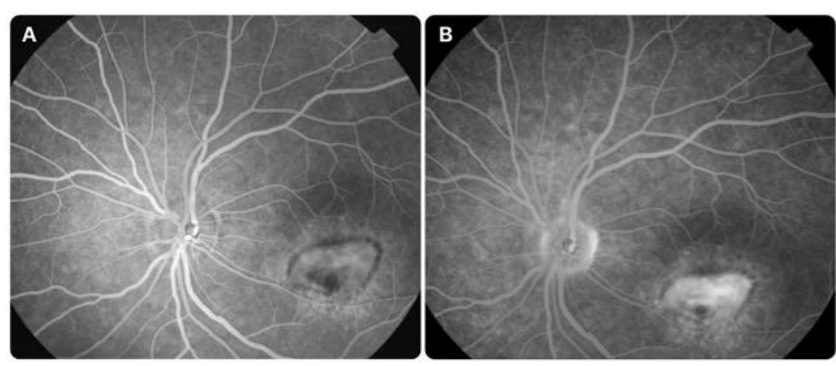

Figure 2: Fluorescein angiogram of the left eye. (A): hyperfluorescence at mid-phase angiography (B): suggestive of pre-epithelial choroidal neovascularization

Left eye Optical Coherence Tomography (OCT) showed a diffuse inhomogeneous area of choroidal hyper-reflectivity suggestive of $\mathrm{CNV}$ associated with a complete shadowing of the underlying retinal pigment epithelium and choroid indicative of a serous pigment epithelium detachment (Figure 3).

Given this clinical presentation of BVMD complicated by type-
Austin J Clin Case Rep - Volume 8 Issue 7 - 2021

ISSN : 2381-912X | www.austinpublishing group.com

Elkhoyaali et al. @ All rights are reserved
Citation: Elkhoyaali A, Jeddou I, Zerrouk R, Khanaouchi N, Mouzari Y, Reda K, et al. Intra-Vitreous Injections of Bevacizumab in Choroidal Neovascularization Complicating Best Vitelliform Macular Dystrophy: Case Report of a Child. Austin J Clin Case Rep. 2021; 8(7): 1220. 


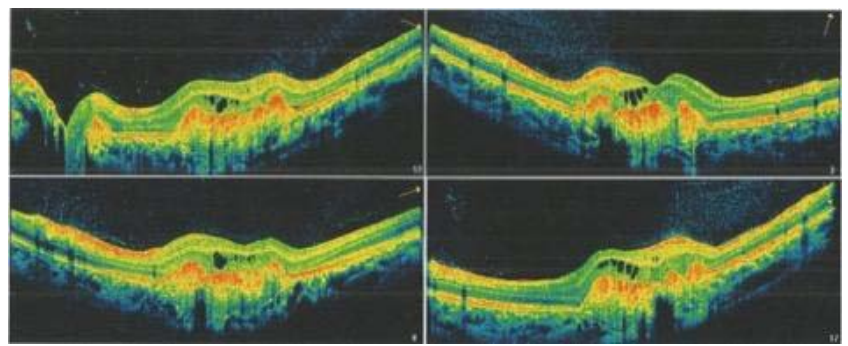

Figure 3: Optical coherence tomography of the left eye before bevacizumab intra-vitreous injections: hyperreflective lesion in front of the pigmentary epithelium compatible with a choroidal neovascularization associated with cystoid fossae of intraretinal oedema and a peripheral subretinal hyporeflective space suggestive of retinal serous detachment.

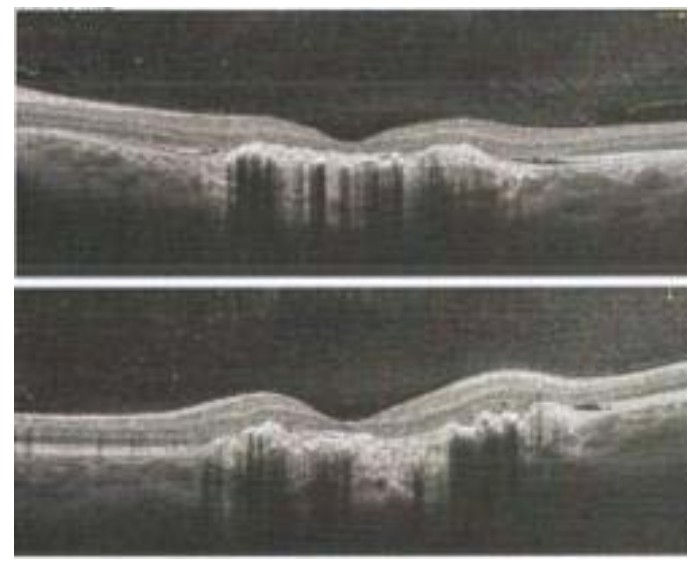

Figure 4: Fundus photography (A) and Optical Coherence Tomography (B) of the left eye five months after starting the treatment: resorption of intraretinal haemorrhage, regression of retinal serous detachment, scarring fragmentation of the vitelline lesion and retraction of the choroidal neovascularization with a hypo-reflective residual blade at the margins of the lesion.

2 choroidal neovascularization, and after parental consent, we performed four intravitreal injections of $1.25 \mathrm{mg}$ of bevacizumab in the left eye under local anaesthesia, at one-month intervals, without any adverse event. As the fourth injection had no positive effect after a good initial response, we decided to stop the injections.

At one month after the fourth injection, visual acuity improved to 20/32 OS and the metamorphopsia disappeared. Intraocular pressure remained normal. Fundus examination showed retraction of the $\mathrm{CNV}$ and resorption of the subretinal haemorrhage. OCT showed resorption of the intraretinal oedema in the left eye with a significant reduction of the SRD but a hyper-reflective layer persisted at the edges of the lesion and the vitelliform material resorbed partially (Figure 4).

At six months after the fourth injection, visual acuity has steadied to 20/32 OS, and after one year of follow-up, the appearance of the macula remained stable with no recurrence of intra- or sub-retinal fluid.

\section{Discussion}

Best vitelliform macular dystrophy is a heredodegenerative maculopathy, usually bilateral, with an autosomal dominant transmission and a variable expressivity and penetrance due to mutations in the BEST1 gene located on chromosome 11 [1]. The product of this gene is the protein Bestrophin-1, a transmembrane protein whose expression is associated with chlorine channels in the Retinal Pigment Epithelium Cells (RPE). Alteration of ions flow would explain both electrophysiological findings and accumulation of a yellow pigment named lipofuscin in the macular subretinal space.

Despite the striking macular lesions exhibited in patients with BVMD that we usually classify into several evolutionary phenotypic stages (pre-vitelliform, vitelliform, pseudohypopic, vitelliruptive, and atrophic stages), the majority maintains good VA over their life, and therefore any sudden visual impairment results from complications. Subretinal neovascularization is an uncommon complication observed in $15 \%$ of all cases predominantly in the later stages [2].

Fundus Autofluorescence (FAF) shows hyper-autofluorescence during the earlier vitelliform stages characteristic of the eggyolk appearance, although in late stages hypo-autofluorescence predominates due to the atrophy of the RPE.

Optical Coherence Tomography (OCT) can be used to localize the vitelliform lesion and evaluate the presence of subetinal fluid or RPE atrophy but FAF remains the gold standard to look after an active Choroidal Neovascularisation (CNV) [3].

This CNV tends to be self-limiting in young people, probably due to the hyper-function of the RPE in the area of the lesion and the production of inhibitors of neovascularization [4].

There is a lot of controversy in the treatment of these CNVs because of the lack of randomized studies comparing the different treatment options:

- Therapeutic abstention in cases of non-significant vision loss or extensive hemorrhage [5].

- Direct laser photocoagulation in cases of extra-foveolar $\mathrm{CNV}$, or photodynamic therapy using Verteporfin [6].

- Anti-Vascular Endothelial Growth Factor (VEGF) therapy, including intravitreal injection of bevacizumab [7], association bevacizumab-triamcinolone or ranibizumab, has been reported more recently as an option for treating $\mathrm{CNV}$.

Bevacizumab, used in our patient, inhibits vascular endothelial growth factor resulting in regression of $\mathrm{CNV}$ and resolution of macular edema. It's used in children on an experimental basis, without pre-established safety consensus, but no prospective study with conclusive results on the potential adverse effects exists currently.

Choroidal neovascularization complicating BVMD generally requires a reduced number of antiangiogenic injections compared to other macular dystrophies [8] associated to an improvement of the visual function without recurrence in most cases, [9] highlighting relatively non-aggressive character of this $\mathrm{CNV}$. Observation findings by Leu et al. [10] and Cakir et al. [8] reported both morphological and functional gains after one single intravitreous injection of bevacizumab and bevacizumab-triamcinolone respectively.

For our case, only four injections enabled complete closure of the neovascular lesion, allowing prompt vision recovery although a fibrotic scar holding a SRD blade persisted, after 12-month follow-up with no recorded side-effects. 


\section{Conclusion}

Though the rapid recovery of visual acuity prompted the authors to recommend the use of antiangiogenic agents in the context of CNV complicating Best disease, larger series with extended follow-up periods are required to define their place in the available therapeutic armamentarium and to assess their possible adverse effects in young patients.

\section{References}

1. Querques G, Zerbib J, Santacroce R, et al. Functional and clinical data of Best vitelliform macular dystrophy patients with mutations in the BEST1 gene. Mol Vis. 2009; 15: 2960-2972.

2. Glacet-Bernard A, Soubrane G, Coscas G. Dégénérescence maculaire vitelliforme de l'adulte. Etude rétrospective d'une série de 85 patients [Macular vitelliform degeneration in adults. Retrospective study of a series of 85 patients]. J Fr Ophtalmol. 1990; 13: 407-420.

3. Lee JY, Lim J, Chung H, Kim JG, Yoon YH. Spectral domain optical coherence tomography in a patient with adult-onset vitelliform dystrophy treated with intravitreal bevacizumab. Ophthalmic Surg Lasers Imaging. 2009; 40: 319321.

4. Ho AC, Yannuzzi LA, Pisicano K, DeRosa J. The natural history of idiopathic subfoveal choroidal neovascularization. Ophthalmology. 1995; 102: 782-789.
5. Querques G, Bocco MC, Soubrane G, Souied EH. Intravitreal ranibizumab (Lucentis) for choroidal neovascularization associated with vitelliform macular dystrophy. Acta Ophthalmol. 2008; 86: 694-695.

6. Gallego-Pinazo R, Dolz-Marco R, Pardo-López D, Arevalo JF, Díaz-Llopis M. Primary intravitreal ranibizumab for adult-onset foveomacular vitelliform dystrophy. Graefes Arch Clin Exp Ophthalmol. 2011; 249: 455-458.

7. Velazquez-Villoria D, Macià Badia C, Rigo Quera J, et al. Bevacizumab intravítreo en neovascularización coroidea asociada a enfermedad de Best [Intravitreal bevacizumab for choroidal neovascularization associated with Best's disease]. Arch Soc Esp Oftalmol. 2014; 89: 405-407.

8. Cakir M, Cekiç O, Yilmaz OF. Intravitreal bevacizumab and triamcinolone treatment for choroidal neovascularization in Best disease. J AAPOS. 2009; 13: 94-96.

9. Eid L, Coste-Verdier V, Rougier MB, Delyfer MN, Korobelnik JF. Imagerie d'un néo-vaisseau choroïdien compliquant une maladie de Best traité par injection intravitréenne de bévacizumab [Imaging of choroidal neovascularization associated with Best's disease treated by intravitreal Bevacizumab]. J Fr Ophtalmol.

10. Leu J, Schrage NF, Degenring RF. Choroidal neovascularisation secondary to Best's disease in a 13-year-old boy treated by intravitreal bevacizumab. Graefes Arch Clin Exp Ophthalmol. 2007; 245: 1723-1725. 